\title{
Ultrafast Pulse Generation in an Organic Nanoparticle-Array Laser
}

\author{
Konstantinos S. Daskalakis, ${ }^{*}$ (이 Aaro I. Väkeväinen, Jani-Petri Martikainen, Tommi K. Hakala, \\ and Päivi Törmä*(i) \\ COMP Centre of Excellence, Department of Applied Physics, Aalto University School of Science, FI-00076 Aalto, Finland
}

\section{Supporting Information}

ABSTRACT: Nanoscale coherent light sources offer potentially ultrafast modulation speeds, which could be utilized for novel sensors and optical switches. Plasmonic periodic structures combined with organic gain materials have emerged as promising candidates for such nanolasers. Their plasmonic component provides high intensity and ultrafast nanoscaleconfined electric fields, while organic gain materials offer fabrication flexibility and a low acquisition cost. Despite reports on lasing in plasmonic arrays, lasing dynamics in these structures have not been experimentally studied yet. Here we demonstrate, for the first time, an organic dye nanoparticlearray laser with more than a $100 \mathrm{GHz}$ modulation bandwidth.

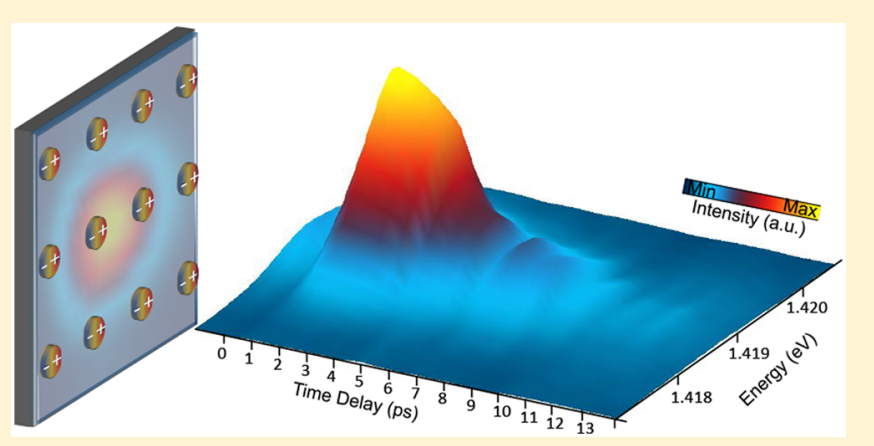
We show that the lasing modulation speed can be tuned by the array parameters. Accelerated dynamics is observed for plasmonic lasing modes at the blue side of the dye emission.

KEYWORDS: Plasmonics, surface lattice resonance, metallic nanoparticle arrays, ultrafast lasing, organic gain

S hrinking the size of a laser device to the nanometer scale leads to two mutually connected advantages over conventional lasers: laser light beyond the diffraction limit and ultrafast modulation bandwidths. ${ }^{1-6}$ Organic lasers are one of the major trends in modern lasing research ${ }^{7-9}$ due to their low cost and ease of fabrication. They can also exhibit high modulation speeds. $^{10,11}$ Here we demonstrate a more than $100 \mathrm{GHz}$ modulation bandwidth for an organic dye nanoparticle-array laser with a high beam directionality, stability, and tunability of the dynamics. The lasing is generated in a system of gold nanoparticles arranged in a periodic array immersed in IR-140 dye solution. The demonstrated ultrafast dynamics are essential for the implementation of nanolasers to practical optoelectronic applications, such as sensing and optical switching, where a subwavelength laser light and ultrafast, low-power lasing operation are required.

Surface lattice resonances (SLRs) are collective optical modes arising from radiative coupling of localized surface plasmon resonances with diffractive orders (DOs) in metal nanoparticle arrays. Such plasmonic lattices provide dispersive modes that can be used to modify the density of states (DOS) and can be brought to strong coupling with emitters. ${ }^{12,13}$ Recent studies have shown that plasmonic structures coupled to organic molecules can shorten the spontaneous decay time of the excited state to the picosecond range, corresponding to a few 100-fold Purcell enhancement in the linear regime. ${ }^{14}$ Lasing in these plasmonic periodic structures combined with an organic gain medium has been reported in the weak ${ }^{14-20}$ and strong ${ }^{21}$ coupling regimes, but a study of the lasing dynamics is notably absent. In previous studies, transient absorption spectroscopy of IR-140 dye emitters near metallic particles was performed and revealed a significant quenching of the excited state lifetime from $\sim 1$ ns to a few tens of picoseconds, ${ }^{14,18}$ but did not give a pulse build-up time and pulse duration. Actual lasing dynamics in plasmonic systems have been studied only in the case of inorganic semiconductor gain media. ${ }^{4}$ There, a $\mathrm{ZnO}$ nanowire was coupled to a metallic mirror, and lasing was generated at the interface of the nanowire/metal. However, direct observation of the lasing beam directionality and momentum-space distribution was inaccessible. Here we present the first extensive investigation of ultrafast lasing dynamics in organic nanoparticle-array lasers; it is also among the first time-resolved studies realized in all types of nanolasers. ${ }^{4,22,23}$ By tuning the periodicity of the nanoparticle array, and thereby the band-edge where lasing occurs, we found a trade-off between having a highly directional intense lasing beam and high modulation speeds.

We consider cylindrical gold nanoparticles patterned in 100 $\mu \mathrm{m} \times 100 \mu \mathrm{m}$ square lattice with periods of $565-585 \mathrm{~nm}$ (Figure 1a) and IR-140 laser dye molecules in a solvent placed on top of the array (Methods). In this configuration, optical confinement is provided by the SLR mode, while gain is supplied by the IR-140 molecules near the gold particles. The SLR dispersion closely follows the DOs of the lattice, which cross at the $\Gamma$-point $\left(k_{\|}=k_{x, y}=0\right)$. Figure $1 \mathrm{~b}$ shows the angleresolved transmission measured along the detection axis $(y$ axis) of a $570 \mathrm{~nm}$ periodicity array and indicates the position of

Received: February 6, 2018

Revised: $\quad$ March 16, 2018

Published: March 20, 2018 


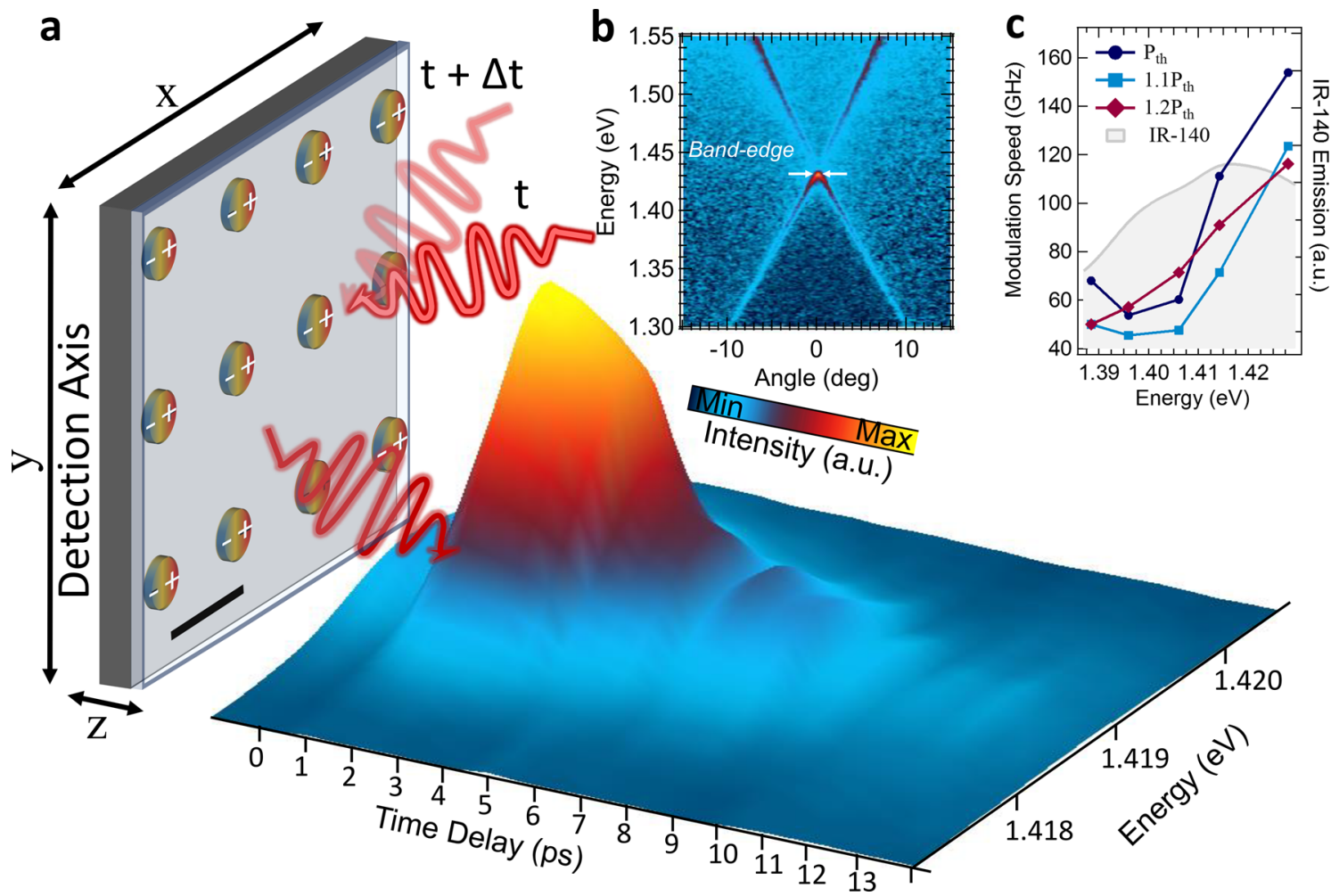

Figure 1. (a) Illustration of the double-pump temporal spectroscopy. On the left is a schematic view of the periodic gold cylindrical-nanoparticle array pumped with a strong pulse at $t$ and probed with a weak pulse, which is delayed by $\Delta t$. The sample is excited by a pulse with electric field polarization along the $x$-axis of the sample ( $x$-polarized dipoles). The scale bar is $570 \mathrm{~nm}$. Only part of the array is depicted in the schematic illustration; the actual arrays are of $100 \mu \mathrm{m} \times 100 \mu \mathrm{m}$ size. On the right is a pseudo-3D contour plot of the experimentally acquired output pulse from an array with a periodicity of $570 \mathrm{~nm}$. At threshold, for this periodicity, we obtain a $0.1 \mathrm{THz}$ modulation bandwidth. (b) Transmission of the sample for mapping out the surface lattice resonance mode dispersion and band-edge. The surface lattice resonance band-edge is located at normal incidence, close to the crossing point of the diffractive orders. (c) Output pulse modulation speed as a function of the band-edge energy (array periods of 565-585 nm). We find an increase of the modulation speed when tuning the band-edge toward the blue side of the IR-140 emission (shown as a grayed-out area).

a

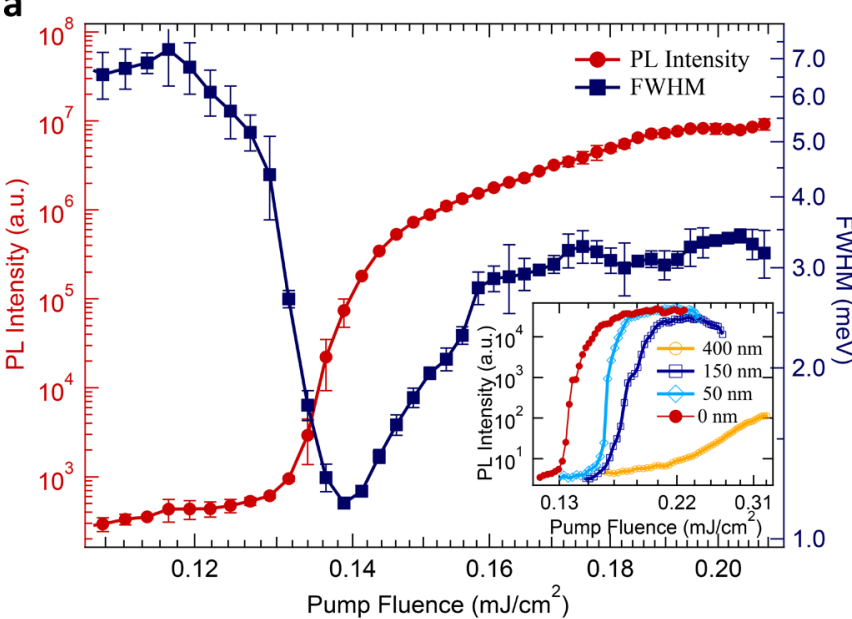

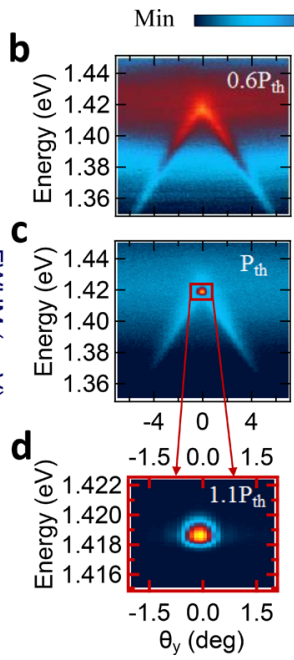
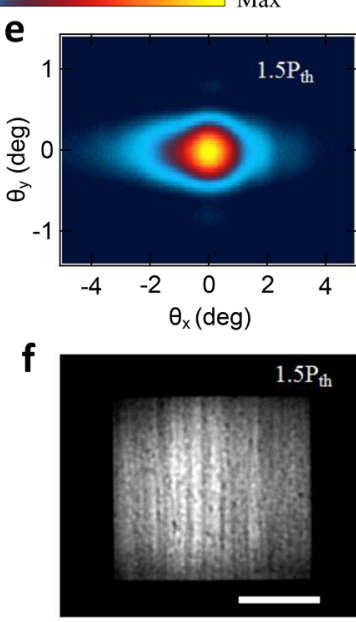

Figure 2. (a) Typical power dependence of the band-edge photoluminescence intensity (red dots) and line width (blue squares) is shown for a gold nanoparticle array of $570 \mathrm{~nm}$ periodicity immersed in $15 \mathrm{mM}$ IR-140 solution. The inset shows the comparison of power dependence graphs when 50, 150, and $400 \mathrm{~nm}$ PVA spacer layer was inserted between the plasmonic array and the IR-140 molecules. (b-d) Photoluminescence below (b) and above threshold (c, d). Note the sharp collapse of the emission to the band-edge. (e, f) Momentum (e) and real-space (f) images of the array lasing emission at $1.5 P_{t h}$. The full-width at half-maximum along $\theta_{y}$ in part e is identical to that in part $\mathrm{d}$, as expected, and slightly broader, $\sim 3^{\circ}$, along $\theta_{x}$. This is attributed to the pump polarization, which generates hot spots at the metallic particles along the $x$-direction: the field distribution and population inversion are thus asymmetric with respect to $x$ and $y$, which determines the lasing polarization ${ }^{20}$ ( $x$-direction) and, furthermore, provides a stronger confinement in $y$-direction $\left(\theta_{y}\right)$. Part $\mathrm{f}$ shows that emission extends over the entire array, albeit with a slightly higher intensity at the center of the array. Scale bar is $50 \mu \mathrm{m}$. 

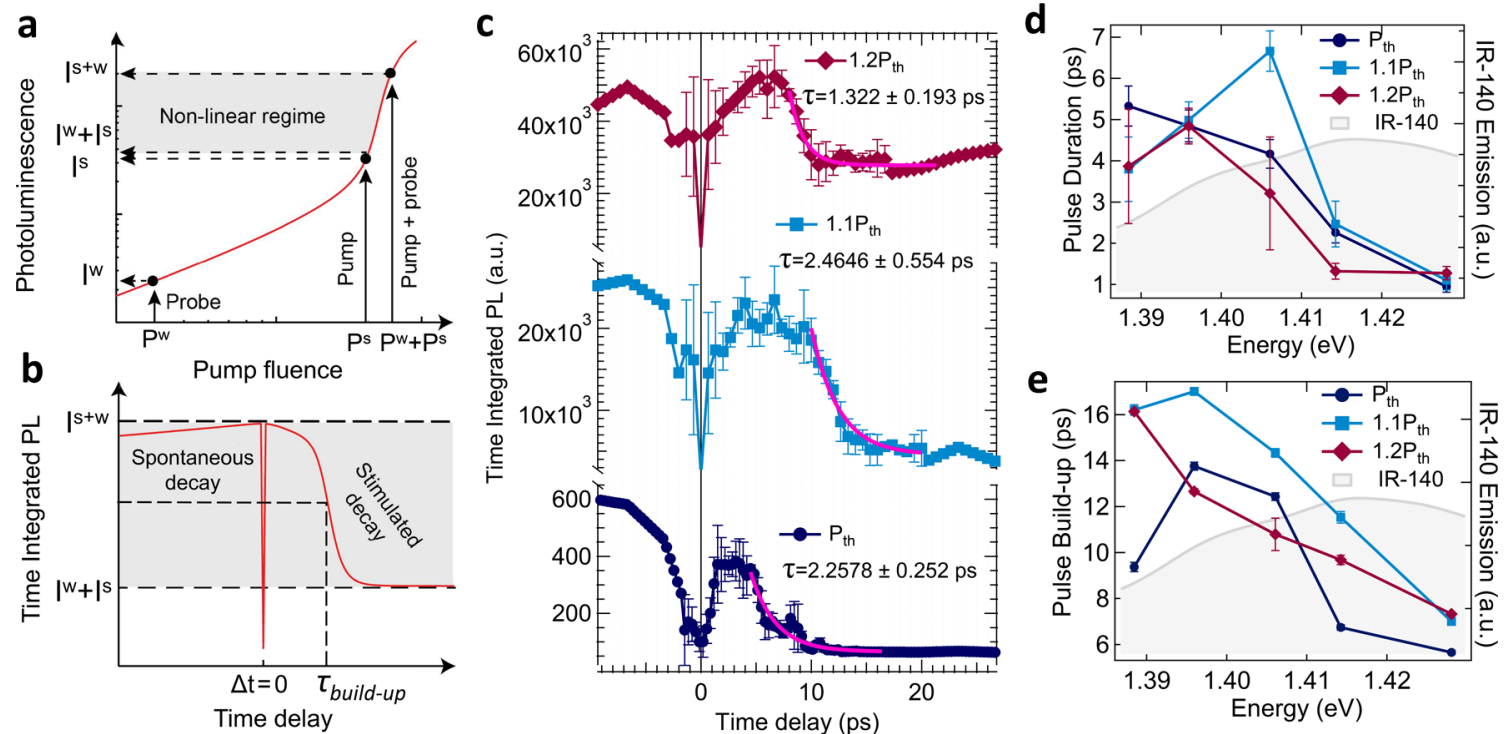

Figure 3. (a, b) Simplified operational principle of the double-pump spectroscopy. (a) Power dependence measurement is used to map the spontaneous $\left(I^{\mathrm{w}}\right)$ and stimulated emission intensities $\left(I^{\mathrm{s}}\right)$ generated by the probe $\left(P^{\mathrm{w}}\right)$ and pump $\left(P^{\mathrm{s}}\right)$ pulses, respectively. When the pump and probe pulses excite the sample at the same time $\left(P^{\mathrm{w}}+P^{\mathrm{s}}\right)$, the output intensity takes a maximum value of $I^{\mathrm{w}+\mathrm{s}}$. When $P^{\mathrm{w}}$ and $P^{\mathrm{s}}$ are well separated in time, the total time-integrated intensity is $I^{\mathrm{w}}+I^{\mathrm{s}}$. (b) By temporally displacing the probe pulse (preceding or following the pump), the spontaneous and stimulated emission (lasing) lifetimes are measured. At zero-delay time, $\Delta t=0$, a dip in the photoluminescence is observed, which is caused by less efficient pumping due to interference fringes when the pulses overlap perfectly in time on the sample (Figure S2). (c) Typical time-integrated photoluminescence for $P_{t h}, 1.1 P_{\text {th }}$ and $1.2 P_{\text {th }}$ for a $570 \mathrm{~nm}$ period array with $100 \mathrm{~nm}$ diameter cylindrical particles polarized along the $x$-axis. The probe was kept at $0.05 P_{t h}$. The output pulse duration was estimated by fitting an exponential decay (magenta line, $\tau$ ) at the positive tail of the doublepump emission. Error bars here are standard deviations from the average value from three different measurement sets, confirming the stability of the lasing. (d) Pulse duration and (e) pulse build-up time dependence on varying the location of the band-edge. We find shortening of both the pulse duration and build-up times when the band-edge is positioned at the blue side of the IR-140 emission (shown in the background of parts $\mathrm{d}$ and e). The modulation speed is defined as the inverse of the sum of the pulse build-up and the pulse duration times. The array periods are 565, 570, 575, 580 , and $585 \mathrm{~nm}$.

the SLR band-edge. Here we used transmission to map out the $\mathrm{E}\left(k_{y}\right)$ SLR dispersion, with $k_{y}=(\omega / c) \sin \theta$ being the in-plane momentum along the $y$-direction, where $\omega$ is the angular frequency, $c$ is the speed of light in a vacuum, and $\theta$ is the angle of incidence along the $y$-axis. The band gap opening at the $\Gamma$ point results from the coupling between counter-propagating SLR modes and increases with a larger metallic particle volume. Note that lasing emerges at the band-edge where feedback required for lasing is provided by the standing wave nature of the band-edge mode. By shrinking the periodicity of the gold particles, the SLR mode dispersion, along with its band-edge location, is shifted to higher energies.

To gain insight into the ultrafast temporal dynamics of the observed lasing, we utilized a recently developed nonlinear alloptical pump-probe technique called double-pump spectroscopy. ${ }^{4}$ In this approach, the time-integrated photoluminescence is recorded (Figure 1a right, pseudo-3D contour plot) when a sample is excited by two laser pulses with identical spectral properties but a different power (Figure la left, sample 3D schematic view): a weak pulse (probe, $P^{\mathrm{w}}$ ), which generates spontaneous emission $\left(I^{\mathrm{w}}\right)$, and a strong pulse (pump, $P^{\mathrm{s}}$ ), which generates laser/nonlinear emission $\left(I^{\mathrm{s}}\right)$. Remarkably, we have measured modulation speeds in the hundreds of $\mathrm{GHz}$ regime by tuning the band-edge to the blue side of the IR-140 emission. This is shown in Figure 1c where the output pulse bandwidth is plotted as a function of the band-edge energy.

We studied the linear and nonlinear emission properties of the lasing as a function of the pump pulse fluence. Figure $2 \mathrm{a}$ shows a power dependence measurement of photoluminescence collected at normal incidence $\left(0^{\circ}, k_{\|}=0\right)$ from an array with $570 \mathrm{~nm}$ periodicity. For an increasing pump fluence, we find that at the threshold of $132 \mu \mathrm{J} / \mathrm{cm}^{2}$, the strong increase of the photoluminescence is accompanied by a collapse in line width, indicative of the increase in temporal coherence. A second plateau of the linear regime is reached at $\sim 150 \mu \mathrm{J} / \mathrm{cm}^{2}$, where the total emission is increased by 3 orders of magnitude, corresponding to a spontaneous emission coupling factor $(\beta$ factor) of $\sim 0.001$. Photobleaching and unstable lasing are common problems in organic semiconductors and dyes. ${ }^{9}$ In addition, the ultrashort and intense pulses used in the high temporal-resolution pump-probe measurements can permanently damage the metallic nanostructures and dye molecules, leading to a false estimation of the threshold and misinterpretation of the temporal dynamics. The standard deviation error bars in our power dependence curves confirm the sample's highly stable emission characteristics, an important parameter for the reliability of our double-pump measurement.

To confirm that the lasing mode is mostly confined in subwavelength spatial regions near the nanoparticles, we introduced a poly(vinyl alcohol) (PVA) film that was used as a spacer (in the $z$-direction) between the IR-140 molecules and metallic particles. We performed power dependence measurements for 50, 150, and $400 \mathrm{~nm}$ PVA spacers (inset in Figure 2a) and observed that a $50 \mathrm{~nm}$ spacer increases the threshold $23 \%$, while there was no lasing at all for a $400 \mathrm{~nm}$ PVA spacer.

The angle-dependent emission is shown below (Figure $2 b$ ), at and above threshold (Figure 2c,d). Below threshold, the emission follows the SLR dispersion that was measured in transmission. At threshold, the emission collapses to the bandedge and is strongly confined in momentum and energy, albeit 
a

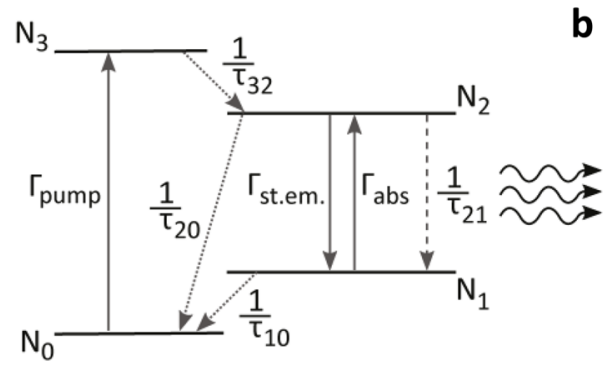

b

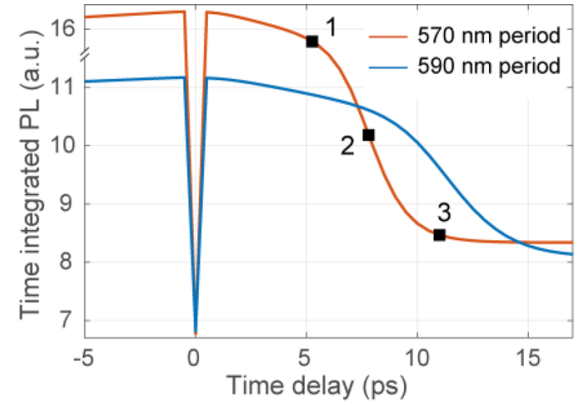

Rate-equation simulation

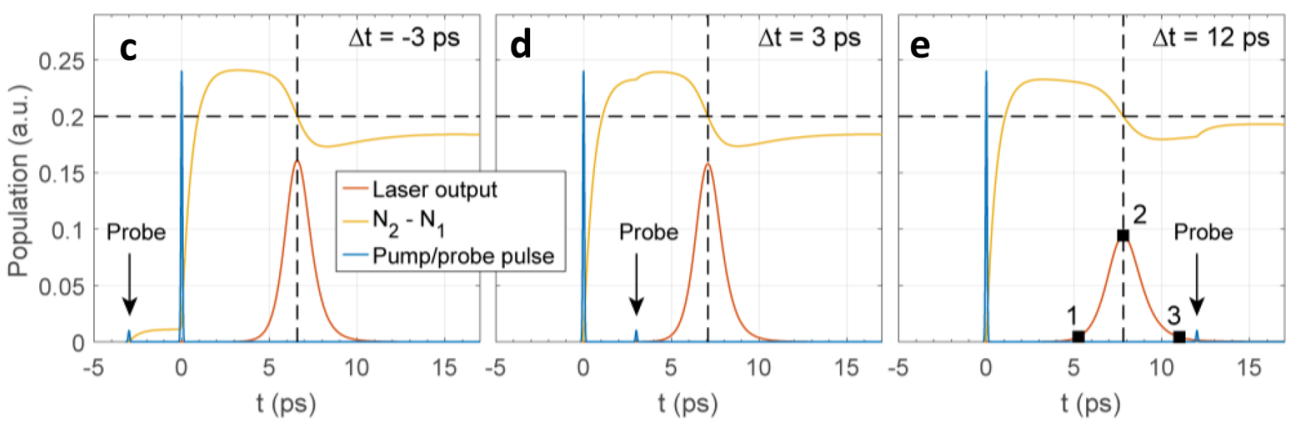

FDTD simulation

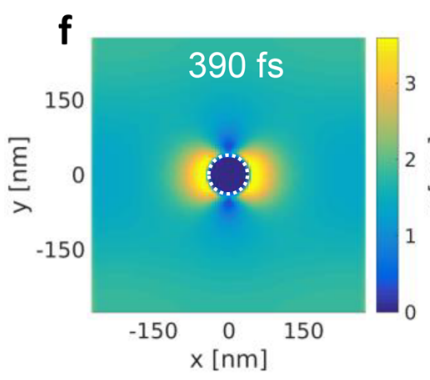

g
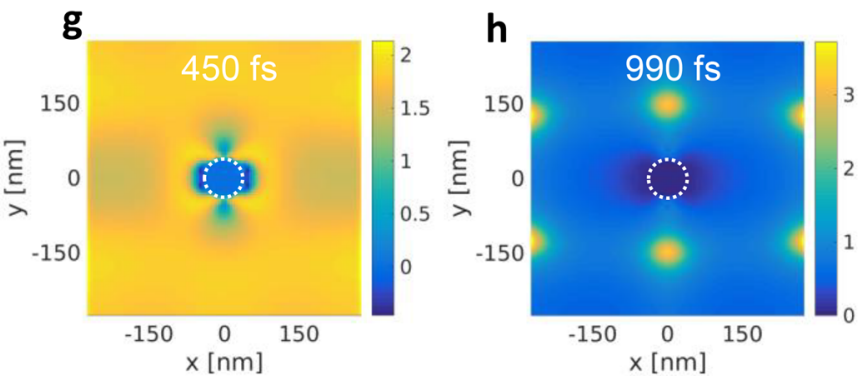

Figure 4. (a) Energy level diagram of the gain medium. (b-e) Rate-equation simulations with a pump and a probe pulse. (b) Typical time-integrated photoluminescence for $1.2 P_{\text {th }}$ with two different parameter choices. The red line corresponds to $570 \mathrm{~nm}$ and blue line to $590 \mathrm{~nm}$ array period. The smaller period results in a shorter SLR lifetime and $\tau_{32}$, and thus faster decay of laser emission is observed (Methods). The probe power was kept at $0.05 P_{t h}$, and the dip at zero-delay time is set manually to represent the interference-caused dip in the experimental data (Figure S2). The three black squares correspond to the temporal locations marked in part e. The laser output peaks at the half-maximum of the time-integrated emission, which we define as the built-up time (point 2). (c-e) Population trajectories corresponding to the parameter choices of the red curve in part b (570 $\mathrm{nm}$ period). Input pulses (blue), population inversion $N_{2}-N_{1}$ (yellow), and output laser pulse (red). The probe pulse, with delay $\Delta t$, is also indicated with arrows for visibility. Dashed horizontal line indicates the threshold value for population inversion and the laser output peaks when the population inversion crosses the threshold value (indicated with a dashed vertical line). (f-h) FDTD simulations of inversion $N_{2}-N_{1}$ in the $x y$ plane for an $x$-polarized dipole for three different time frames: (f) before lasing but after the pump pulse ( $390 \mathrm{fs}),(\mathrm{g})$ when lasing starts (450 fs), and (h) after the generated output pulse (990 fs). The color scale is in $\mathrm{mM}$. At longer times, the peaks in gain match the locations of the vanishing electric field in the array; see the Supporting Information, Figure S7. The FDTD simulations in parts $\mathrm{f}-\mathrm{h}$ were performed with a single pump pulse with a fluence of $I=0.03 \mathrm{~mJ} / \mathrm{cm}^{2}$. For clarity, we have marked the boundary of the nanoparticle with a white dashed line. The difference in time scales between the rate-equation and FDTD simulations comes mainly from a difference in the pumping level. In the FDTD simulations, the pump fluence was set much higher above the threshold level such that the output pulse occurs in a feasible time frame. Also, the rate-equation model shows considerably accelerated dynamics if the pump level is increased to multiple times the threshold (Figure S13). The main purpose of the FDTD simulations is to show the spatial dependence of the population inversion and how it corresponds to the field hot spots (Figure S7).

with some weak uncoupled dye emission. Above threshold, only strong emission from the band-edge is observed. The excellent laser beam quality is shown in Figure $2 \mathrm{~d}$ where its spectral full-width at half-maximum (fwhm) is $\sim 1 \mathrm{meV}$, and the beam is confined within $\sim 1^{\circ}$. The directionality and strong confinement of the laser beam is shown in the $2 \mathrm{D}$ momentumspace image in Figure 2e. The real-space image of the lasing emission is shown in Figure $2 \mathrm{f}$. When the band-edge was located on the red side of the dye emission, we see a decrease of the lasing threshold (Figure S4b), similar to what has been previously reported for all of the dye-based vertical cavity surface emitting lasers (VCSELs). ${ }^{9}$ This is due to optimization between having the maximum emission at the minimum absorption in a Stokes-shifted gain material.

Figure 3a,b depicts the operational principle of the doublepump spectroscopy; ${ }^{4}$ see Methods for more details. At negative time delays, $\Delta t<0$, the weak probe pulse arrives first and that alone cannot create a sufficient population inversion to start lasing action. The population decays slowly through spontaneous decay channels (radiative and nonradiative) until the strong pump pulse arrives and triggers the laser pulse. The behavior of the output intensity for negative delays can be used for measuring the spontaneous decay time, in our case 180 ps (Figure S5). At positive delays, $\Delta t>0$, the pump generates a 
population inversion that exceeds the threshold value and a lasing pulse is triggered. The probe can contribute to lasing intensity only if it arrives before the generated laser pulse ends. If the probe arrives later, the emitters it excites decay through spontaneous channels and add only a small contribution to the time-integrate output signal, which is a fraction of spontaneous emission determined by the $\beta$-factor. To summarize, by varying the delay between pump and probe pulses, we can measure both the Purcell enhancement of spontaneous emission caused by the structure (negative delays) and the dynamics of the lasing pulse (positive delays). Utilizing the power dependence mapping of Figure $2 \mathrm{a}$, the probe was set and kept at $0.05 P_{\text {th }}$ throughout the measurement. We chose the value $0.05 P_{\text {th }}$ as an optimal trade-off between constricting any parametric modification of the lasing dynamics ${ }^{24}$ and measurable timeintegrated emission response.

Since we are interested in the dynamics of the nonlinear regime and due to the very sharp nonlinear response of the laser device, we measured three different pump regimes, namely, $P_{t h}, 1.1 P_{t h}$, and $1.2 P_{t h}$. Figure $3 \mathrm{c}$ shows the timeintegrated lasing emission at the band-edge for varying the delay of the probe pulse. For small negative time delays $(\Delta t<$ 0 ), we see a high value of photoluminescence, as expected when the pump and probe powers sum up to produce $I^{\mathrm{s}+\mathrm{w}}$, and an interference-caused tip at $\Delta t=0$. For positive time delays $(\Delta t>$ $0)$, the time-integrated emission reaches again $I^{s+w}$, which is then followed by a rapid decay to $I^{\mathrm{s}}+I^{\mathrm{w}}$. In Figure 3c, we plot the time-integrated emission from an array with band-edge tuned at $1.414 \mathrm{eV}$. One of the most startling features of our system is the strong dependence of the dynamics on the bandedge location. As we tune the band-edge onto the blue side of the IR-140 emission, an increase of the lasing modulation speed is observed.

Our device is essentially an optically pumped gain-switched laser. ${ }^{25}$ First the population inversion is excited well above the threshold with the strong pump pulse. Then, spontaneous emission triggers lasing action, and the upper laser level rapidly depletes by stimulated emission. The population inversion is burned down much below the threshold value, and an output pulse is generated. In the double-pump experiment, the probe pulse reveals the delay between zero-time, the instance when the pump pulse turns the laser on, and the end of laser emission. We can extract the pulse duration (fwhm of the generated pulse) by fitting an exponential function on the positive tail of the time-integrated emission (Figure 3d). We estimate the pulse build-up time (Figure $3 \mathrm{e}$ ) to be the delay between zero-time and the half-maximum of the timeintegrated photoluminescence (Figure $3 \mathrm{~b}$ ). The estimate is based on a rate-equation model (Figure 4) and is explained below. The laser modulation bandwidth is determined by the delay between turning the laser on (pump pulse) and the end of laser emission. In our case, the bandwidth is an inverse of the sum of the pulse build-up time and the pulse duration.

We also studied the lasing dynamics for dipole polarization along the $y$-axis instead of $x$ (Figure S6a-c), and of arrays with 80 and $100 \mathrm{~nm}$ particle diameter, and periodicities of 560-590 $\mathrm{nm}$ (Figure S6d,e). The band-edge of arrays with $80 \mathrm{~nm}$ particles can be tuned to be at the deep-blue side of the IR-140 emission spectrum. Interestingly, at these higher energies, the modulation speed reaches the $\mathrm{THz}$ regime; however, this record speed was seen only for a sample with $y$-polarized dipoles. See the Supporting Information, Figure S6.
We have measured altogether 30 array samples with periodicities from 565 to $585 \mathrm{~nm}$ and particle sizes from 80 to $120 \mathrm{~nm}$, of which all showed clear lasing. For pump fluences up to $11 \mathrm{~mJ} / \mathrm{cm}^{2}$, we did not observe degradation of the particles or arrays. For pumping powers larger than $0.32 \mathrm{~mJ} /$ $\mathrm{cm}^{2}$, the lasing dye shows photobleaching, but when the pump is reduced below $0.32 \mathrm{~mJ} / \mathrm{cm}^{2}$, lasing is recovered due to continuous refreshing of the solvent/dye solution by natural circulation.

To comprehend the dynamics of the lasing action and to extract the build-up time and pulse duration from the experimental time-integrated signal, we use a rate-equation model with a four-level gain medium. The schematic view of the model is shown in Figure $4 \mathrm{a}$ and can be described as follows: a Gaussian pump excites a substantial fraction of population into the highest level $\left(N_{3}\right)$, which then decays into the upper laser level $\left(N_{2}\right)$, initiating the inversion $\left(N_{2}-N_{1}\right)$ for the laser transition. Similarly to the experimental configuration (Figure 3a,b), we use a probe pulse, which does not generate a population inversion sufficient for lasing, but it contributes in the output pulse power depending on its relative location in time. Time-integrated photoluminescence is recorded, similar to the experiments, as a function of probe delay, as shown in Figure $4 \mathrm{~b}$. The trajectories of input pulses, population inversion between upper and lower laser level $\left(N_{2}-N_{1}\right)$, and generated lasing output pulse are presented in Figure $4 \mathrm{c}-\mathrm{e}$ for delay times of -3 ps, 3 ps, and 10 ps.

Mapping from time-integrated photoluminescence to the laser pulse generation is shown in Figure 4 b,e. First, the pulse build-up time can be defined as the time delay between zerotime and half-maximum of the time-integrated signal (point 2). It corresponds to the maximum lasing output. Second, the point where the decay starts (point 1 ) and where the decay ends (point 3) confines the output pulse in time. Fitting an exponential function to the slope gives a good estimate for the pulse duration.

The rate-equation simulations help reveal the origin of the acceleration of the dynamics when tuning the band-edge to the blue side of the emission. The pulse duration and build-up times in the simulations depend on the spontaneous emission factor $\beta$ and on the lifetimes $\tau_{21}, \tau_{32}$, and $\tau_{\text {cav }}$, where $\tau_{\text {cav }}$ is the SLR mode lifetime. A thorough analysis of the modulation speed in this four-dimensional parameter space is presented in Figure S12. It reveals that, for parameter ranges relevant for our experiments, the cavity (SLR mode) lifetime is the dominant factor. Therefore, the shortening of the SLR mode lifetime at higher energies is likely the main explanation for the observed faster dynamics.

In FDTD simulations, we can include the spatial dependence of the fields, which the rate-equation model does not do (Supporting Information, Method 2), and obtain a microscopic picture of the lasing action. Notably, while inversion is quite homogeneous away from the particles, it can be strongly inhomogeneous close to it (Figure $4 \mathrm{f}-\mathrm{h}$ ). This reflects the strong position dependence of the electric field close to the metallic particles. In the FDTD simulations, the lasing pulse appears earlier than in measurements, which is due to a relatively large uncertainty in the parameters of the four-level model for the gain media and the threshold. Here, we are most probably high above the threshold, as also the rate-equation model generates a laser pulse within a picosecond when the pump is set at $8 P_{t h}$ (Figure S13). The faster pulse dynamics for 
larger pump fluences corresponds to an increasing line width of the lasing emission in the frequency domain.

Plasmonic lattices are a versatile system where a variety of different geometries, superlattices, nanoparticle shapes, and gain materials can be easily realized. This has already lead to demonstrations of strong coupling, ${ }^{13}$ single- and multimode lasing in bright and dark modes both in the weak ${ }^{14,19,20}$ and strong $^{21}$ coupling regimes, as well as very recently BoseEinstein condensation. ${ }^{26}$ Our demonstration of ultrafast, tunable dynamics in plasmonic lattices therefore has ramifications for a wide range of new fundamental phenomena and applications.

To summarize, we have experimentally demonstrated a 100 $\mathrm{GHz}$ modulation bandwidth of lasing in an organic gain medium surrounding a plasmonic nanoparticle lattice. Shortening of both the pulse build-up and duration times was achieved by tuning the band-edge on the blue side of the gain medium emission maximum. Rate-equation simulations were used for describing the dynamics, and FDTD simulations revealed the nontrivial spatial dependence of the field profiles during the lasing action. Both simulations confirm the ultrafast dynamics for realistic model parameters. Our results show that organic gain material, combined with a plasmonic nanostructure, can provide ultrafast lasing dynamics. The combination of high modulation speeds, small footprint, and low acquisition cost provided by the organic gain suggests that this type of laser is promising for all-optical switching and sensing. In this context, the stability and tunability of our laser devices are highly important characteristics.

Methods. Sample Fabrication. The gold nanoparticle arrays were fabricated on borosilicate glass slides with electron-beam lithography. To couple the dye molecules to the gold arrays, a $15 \mathrm{mM}$ solution of IR-140 dye in 1:2 dimethyl sulfoxide/benzyl alcohol (DMSO/BA) was deposited on the array substrate. This mixing ratio of DMSO/BA was chosen to match the refractive index of the borosilicate substrate. To seal the IR-140 solution on the substrate, an identical borosilicate glass slide was placed on top. In this configuration, the dye solution creates a uniform film of $\sim 10 \mu \mathrm{m}$, which surrounds the arrays and bonds the glass substrates. Nanoparticle arrays can support both dark and bright mode lasing. ${ }^{19}$ Here our focus is the dynamics, and we wish to avoid complexity arising from the multimode operation. Therefore, we have chosen particle size and other parameters such that they favor bright mode lasing. ${ }^{19}$

Experimental Setup and Time-Integrated Double-Pump Spectroscopy. A schematic view of the optical setup is shown in Figure S1. Angle-resolved transmission and photoluminescence were obtained by collecting the transmitted/emitted light with an objective $(10 \times, 0.3 \mathrm{NA})$. We focused the back-focal plane image of the objective to the entrance slit of the spectrometer. The spectrometer slit stands in vertical orientation ( $y$-axis), and therefore each position along the $y$ axis of the CCD corresponded to a particular angle of transmitted/emitted light $\left(\theta_{y}\right)$, which maps to an in-plane momentum of optical modes on the sample. A beam splitter was used to direct part of the image to a second CCD camera for far-field real-space or 2D-momentum-space imaging. LabVIEW software was developed in house to automatize both power dependence and double-pump measurements and to allow acquisition of real-space and momentum-space sample images simultaneously. We excited the sample above threshold with a $70 \mathrm{fs}$ pump pulse. The time-integrated nonlinear emission was generated with a weak probe pulse that had the same spectral properties as the pump. The angle of incidence, for both pump and probe pulses, was $\sim 45^{\circ}$, and the spots were elliptical with an area of $0.1 \mathrm{~mm}^{2}$. Note that the spot size was chosen to be ten times larger than the square arrays to ensure spatial overlap between the pump and the probe throughout the displacement range of the motorized probe pulse mirror stage.

The double-pump spectroscopy, ${ }^{4}$ Figure $3 \mathrm{a}, \mathrm{b}$, operates as follows. At negative time delays, $\Delta t<0$, the probe proceeds the pump and excites molecules to the upper laser level. However, the population inversion is insufficient to trigger lasing action. Instead, the excited population decays through spontaneous decay channels (radiative and nonradiative), considerably slower. If the negative time delay is larger than the spontaneous emission time, one observes the output for essentially independent pulses, $I^{\mathrm{w}}+I^{\mathrm{s}}$. If the negative time delay is smaller than the spontaneous decay time of the molecule, the population inversion created by the weak probe will hold until the strong pump arrives, and thus the probe and pump powers effectively sum up, $P^{\mathrm{w}}+P^{\mathrm{s}}$, and produce an output intensity $I^{\mathrm{w}+\mathrm{s}}$, which is larger than $I^{\mathrm{w}}+I^{\mathrm{s}}$ due to the strong nonlinearity around the threshold power. Therefore, the behavior of the output intensity for negative delays can be used for measuring the spontaneous decay time. The situation is completely different at positive delays, $\Delta t>0$. The population inversion, generated by the pump, exceeds the lasing threshold value, resulting in stimulated (fast) laser pulse emission. The population inversion decays quickly, and the probe can only contribute to the lasing pulse if it arrives before the output pulse is emitted. In other words, the pump and probe powers effectively sum up and give $I^{\mathrm{w}+\mathrm{s}}$ only if the probe arrives during the laser pulse generation. Otherwise the time-integrated output is $I^{\mathrm{w}}+I^{\mathrm{s}}$. Consequently, we can directly measure the lasing dynamics (positive delays) and Purcell enhancement (negative delays) by simply varying the delay between pump and probe pulses. Note that the standard deviation bars in power dependence and double-pump spectroscopy represent the average of 3 or 4 measurements of the same array. The formula that we used to calculate the standard deviation is $\sigma=\sqrt{\Sigma\left(Y_{i}-V_{\text {average }}\right) /\left(V_{\text {npnts }}-1\right)}$, where $V_{\text {average }}$ is the average value, $V_{\text {npnts }}$ is number of measurements, and $Y_{i}$ is the value of each measurement.

Rate-Equation Model. The material parameters for rateequation model were obtained as follows. In ref 27 , the radiative decay time (spontaneous emission lifetime) of level 2 is $\tau_{21}=5.1 \mathrm{~ns}$, the vibrational relaxation times $\tau_{32}=0.7 \mathrm{ps}$ and $\tau_{10}=4 \mathrm{ps}$, and the nonradiative decay time $\tau_{20}=240 \mathrm{ps}$ (Figure $4 \mathrm{a})$. From these, we used $\tau_{10}$ and $\tau_{20}$ directly because there is no particular reason to assume that these vibrational relaxation times would change in our setting. However, we calculated the spontaneous emission lifetime based on the measured spontaneous decay time, 180 ps, which includes the contribution of both radiative and nonradiative decay. By assuming that the Purcell effect enhances only the radiative decay rate, we obtain $\tau_{21}=720$ ps. We neglect the enhancement of the nonradiative rate because in the context of a four-level model, the nonradiative transition $\left(\tau_{20}\right)$ accounts for all complicated molecular relaxation processes such as vibrational relaxations, which would otherwise be beyond the four-level model. Purcell effect is a resonant process, ${ }^{28}$ and we assume that the nonradiative transition (containing one or more steps) is not in resonance with the lasing mode (SLR). 
Fluorescence quenching is significantly very close (a few nanometers) to the metal, ${ }^{29,30}$ and the fraction of quenched emitters within the total lasing mode volume is a minute. Finally, $\tau_{32}$ should be the same order of magnitude as the literature value, but it can also be shorter because, when we tune the lasing energy (the band-edge) toward higher energies (blue side of the dye emission), the molecule does not need to relax as far in the vibrational manifold before emitting a photon. Thus, $\tau_{32}$ in the four-level model can be effectively shorter, which can enhance the dynamics.

The lasing mode is assumed to have a plasmonic character, and its lifetime corresponds to the measured SLR lifetime. We found that the line width of the SLR mode slightly broadens when moving toward higher energies, which is due to the increase of the plasmonic component of the SLR. The quality factor of the SLR at 590 and $570 \mathrm{~nm}$ period gives $Q=254$ and $Q=182$, respectively. These convert to lifetimes of $\tau_{\mathrm{cav}}=121 \mathrm{fs}$ and $\tau_{\text {cav }}=84 \mathrm{fs}$. The lasing dynamics in the rate-equation model is very sensitive to the lasing mode (or cavity) lifetime, and the shorter lifetime with a shorter period (band-edge in higher energy) leads to faster built-up time and a shorter pulse duration. We have simulated the lasing action with two different parameter choices: (1) $\tau_{\text {cav }}=121 \mathrm{fs}, \tau_{32}=700 \mathrm{fs}$ and (2) $\tau_{\text {cav }}=$ $84 \mathrm{fs}, \tau_{32}=577 \mathrm{fs}$. The first choice corresponds to a $590 \mathrm{~nm}$ period with a band-edge at $1.39 \mathrm{eV}$, and second one corresponds to a $570 \mathrm{~nm}$ period with a band-edge at 1.43 $\mathrm{eV}$. The change of $\tau_{32}$ is proportional to the energy difference from the pump (at $800 \mathrm{~nm}, 1.55 \mathrm{eV}$ ) to the band-edge. Faster dynamics giving a higher modulation speed was obtained for the second parameter set as seen in Figure $4 \mathrm{~b}$. The major contribution to faster dynamics yields from the change in the mode lifetime. We have performed a systematic analysis of the modulation speed in the four-dimensional parameter space of $\beta$, $\tau_{21}, \tau_{32}$, and $\tau_{\text {cav }}$; see Figure S12. The employed rate-equations are described in detail in the Supporting Information, Method 1.

FDTD Model. Our FDTD model for lasing adapts a semiclassical lasing model, ${ }^{25}$ with a four-level gain medium, to the array structure (Supporting Information, Method 2). For the gain medium, we used lifetimes $\tau_{30}=1 \mathrm{~ns}, \tau_{32}=0.7 \mathrm{ps}, \tau_{10}=$ $4 \mathrm{ps}$, and $\tau_{21}=720 \mathrm{ps}$. Here we used the enhanced radiative decay rate for $\tau_{21}$ rather than the value $(5.1 \mathrm{~ns})$ in a vacuum.

We cover one side of the array with a $200 \mathrm{~nm}$ thick layer of gain material with a concentration of $15 \mathrm{mM}$. We use perfectly matched layer (PML) boundaries along the $z$-axis and periodic boundary conditions along the $x$ - and $y$-directions. Here we also assume that the solutions are symmetric in the $x y$-directions. The system is excited with plane-wave pulses that travel orthogonally to the array plane and generate $x$-polarized dipoles (Figure S7). Note that for the FDTD simulations, we present the dynamics following a single pump pulse, not pump-probe experiment simulations as for the rate-equation simulations. This is because the main purpose of the FDTD simulations is to demonstrate the highly inhomogeneous and nontrivial spatial dependence of the fields during the lasing action.

\section{ASSOCIATED CONTENT}

\section{S Supporting Information}

The Supporting Information is available free of charge on the ACS Publications website at DOI: 10.1021/acs.nanolett.8b00531.
Additional figures of experimental setup, double-pump resolution, band-edge mode lifetime, lasing power dependence and dynamics for $y$-polarized dipoles, spontaneous emission lifetime, electric filed magnitude, SEM image, power dependence spectra, power dependence of IR-140, directionality at deep-blue region, sensitivity analysis of the rate-equation model, and rateequation simulation at $8 \mathrm{P}_{\text {th }}$ as well as methods for rateequation and FTDT modeling (PDF)

\section{AUTHOR INFORMATION}

\section{Corresponding Authors}

*E-mail: konstantinos.daskalakis@aalto.fi.

*E-mail: paivi.torma@aalto.fi.

\section{ORCID}

Konstantinos S. Daskalakis: 0000-0002-3996-5219

Päivi Törmä: 0000-0003-0979-9894

\section{Author Contributions}

K.S.D. and A.I.V. conducted the measurements. A.I.V. fabricated the samples and did the rate-equation modeling. J.P.M. performed the FDTD simulations. All authors discussed the results. K.S.D., P.T., and A.I.V. wrote the manuscript together with all authors. P.T. supervised the project.

\section{Notes}

The authors declare no competing financial interest.

\section{ACKNOWLEDGMENTS}

This work was supported by the Academy of Finland through its Centres of Excellence Programme (2012-2017), under project nos. 284621, 303351, and 307419, and by the European Research Council (ERC-2013-AdG-340748-CODE). This article is based upon work from COST Action MP1403 Nanoscale Quantum Optics, supported by COST (European Cooperation in Science and Technology). Part of the research was performed at the Micronova Nanofabrication Centre, supported by Aalto University. The Triton cluster at Aalto University was used for the computations. K.S.D. acknowledges financial support by a Marie Skłodowska-Curie Action (H2020MSCA-IF-2016, project id 745115) and thanks T. S. Sidiropoulos for helpful discussions on the double-pump experiment.

\section{REFERENCES}

(1) Bergman, D. J.; Stockman, M. I. Phys. Rev. Lett. 2003, 90, 027402.

(2) Oulton, R. F.; Sorger, V. J.; Zentgraf, T.; Ma, R.-M.; Gladden, C.; Dai, L.; Bartal, G.; Zhang, X. Nature 2009, 461, 629-632.

(3) Ma, R.-M.; Oulton, R. F.; Sorger, V. J.; Zhang, X. Laser \& Photonics Reviews 2013, 7, 1-21.

(4) Sidiropoulos, T. P. H.; Röder, R.; Geburt, S.; Hess, O.; Maier, S. a.; Ronning, C.; Oulton, R. F. Nat. Phys. 2014, 10, 870-876.

(5) Hill, M. T.; Gather, M. C. Nat. Photonics 2014, 8, 908-918.

(6) Pan, S. H.; Gu, Q.; Amili, A. E.; Vallini, F.; Fainman, Y. Optica 2016, 3, 1260.

(7) Bradley, D. D. C. Nature 1996, 382, 671-671.

(8) Daskalakis, K. S.; Maier, S. A.; Murray, R.; Kéna-Cohen, S. Nat. Mater. 2014, 13, 271-8.

(9) Kuehne, A. J. C.; Gather, M. C. Chem. Rev. 2016, 116, 1282312864.

(10) Samuel, I. D. W.; Turnbull, G. A. Chem. Rev. 2007, 107, 127295.

(11) Clark, J.; Lanzani, G. Nat. Photonics 2010, 4, 438-446.

(12) Törmä, P.; Barnes, W. L. Rep. Prog. Phys. 2015, 78, 013901. 
(13) Väkeväinen, A. I.; Moerland, R. J.; Rekola, H. T.; Eskelinen, A.P.; Martikainen, J.-P.; Kim, D.-H.; Törmä, P. Nano Lett. 2014, 14, $1721-1727$.

(14) Zhou, W.; Dridi, M.; Suh, J. Y.; Kim, C. H.; Co, D. T.; Wasielewski, M. R.; Schatz, G. C.; Odom, T. W. Nat. Nanotechnol. 2013, 8, 506-511.

(15) van Beijnum, F.; van Veldhoven, P. J.; Geluk, E. J.; de Dood, M. J. A.; t Hooft, G. W.; van Exter, M. P. Phys. Rev. Lett. 2013, 110, 206802.

(16) Meng, X.; Liu, J.; Kildishev, A. V.; Shalaev, V. M. Laser Photon. Rev. 2014, 8, 896-903.

(17) Schokker, A. H.; Koenderink, A. F. Phys. Rev. B: Condens. Matter Mater. Phys. 2014, 90, 155452.

(18) Yang, A.; Hoang, T. B.; Dridi, M.; Deeb, C.; Mikkelsen, M. H.; Schatz, G. C.; Odom, T. W. Nat. Commun. 2015, 6, 6939.

(19) Hakala, T. K.; Rekola, H. T.; Väkeväinen, A. I.; Martikainen, J. P.; Nečada, M.; Moilanen, A. J.; Törmä, P. Nat. Commun. 2017, 8, 13687.

(20) Wang, D.; Yang, A.; Wang, W.; Hua, Y.; Schaller, R. D.; Schatz, G. C.; Odom, T. W. Nat. Nanotechnol. 2017, 12, 889.

(21) Ramezani, M.; Halpin, A.; Fernández-Domínguez, A. I.; Feist, J.; Rodriguez, S. R.-K.; Garcia-Vidal, F. J.; Gómez Rivas, J. Optica 2017, 4, $31-37$.

(22) Röder, R.; Sidiropoulos, T. P. H.; Tessarek, C.; Christiansen, S.; Oulton, R. F.; Ronning, C. Nano Lett. 2015, 15, 4637-4643.

(23) Cuerda, J.; García-Vidal, F. J.; Bravo-Abad, J. ACS Photonics 2016, 3, 1952-1960.

(24) Savvidis, P.; Baumberg, J.; Stevenson, R.; Skolnick, M.; Whittaker, D.; Roberts, J. Phys. Rev. Lett. 2000, 84, 1547-1550.

(25) Siegman, A. E. Lasers; University Science Books, 1986.

(26) Hakala, T. K.; Moilanen, A. J.; Väkeväinen, A. I.; Guo, R.; Martikainen, J.-P.; Daskalakis, K. S.; Rekola, H. T.; Julku, A.; Törmä, P. Bose-Einstein Condensation in a Plasmonic Lattice. 2018, arXiv:1706.01528v2. Nature Physics, in press. https://arxiv.org/abs/ 1706.01528 .

(27) Sperber, P.; Spangler, W.; Meier, B.; Penzkofer, A. Opt. Quantum Electron. 1988, 20, 395-431.

(28) Sauvan, C.; Hugonin, J. P.; Maksymov, I. S.; Lalanne, P. Phys. Rev. Lett. 2013, 110, 237401.

(29) Anger, P.; Bharadwaj, P.; Novotny, L. Phys. Rev. Lett. 2006, 96, 113002 .

(30) Novotny, L.; Hecht, B. Principles of Nano-Optics; Cambridge University Press: Cambridge, 2006. 